\title{
Sphingosine 1-phosphate induces platelet/endothelial cell adhesion molecule-1 phosphorylation in human endothelial cells through $\mathrm{CSrC}$ and $\mathrm{Fyn}^{\text {th }}$
}

\author{
Yu-Ting Huang ${ }^{b}$, Shee-Uan Chen ${ }^{c}$, Chia-Hong Chou ${ }^{c}$, Hsinyu Lee ${ }^{a, b, *}$ \\ a Department of Life Science, National Taiwan University, No 1, Sec. 4, Roosevelt Rd., Taipei, 10617, Taiwan, ROC \\ b Institute of Zoology, National Taiwan University, No 1, Sec. 4, Roosevelt Rd., Taipei, 10617, Taiwan, ROC \\ c Department of Obstetrics and Gynecology, National Taiwan University Hospital, No 7, Chung-Shan South Rd., Taipei, 10002, Taiwan, ROC
}

\section{A R T I C L E I N F O}

\section{Article history:}

Received 23 January 2008

Received in revised form 21 March 2008

Accepted 7 April 2008

Available online 18 April 2008

\section{Keywords:}

Sphingosine 1-phosphate

PECAM-1

cSrc

Fyn

HUVEC

\begin{abstract}
A B S T R A C T
Sphingosine 1-phosphate (S1P) is a multifunctional phospholipid which acts through a specific family of G protein-coupled receptors. Platelet/endothelial cell adhesion molecule-1 (PECAM-1) form trans-homophilic binding at lateral cell border. Upon stimulation, its cytoplasmic tyrosine residues could be phosphorylated and interact with various downstream signaling molecules. In this study, we demonstrated that S1P induced PECAM-1 tyrosine phosphorylation in human umbilical cord vein cells (HUVECs). By pharmacological inhibitors, it was suggested that $G_{i}$ and Src family kinases were involved in PECAM-1 phosphorylation. Moreover, cSrc and Fyn siRNA significantly suppressed S1P-induced PECAM-1 phosphorylation. These results suggested that S1P-induced PECAM-1 phosphorylation through $\mathrm{G}_{\mathrm{i}}$ and subsequent $\mathrm{CSrc}$ and Fyn. Our findings provide further understanding of S1P and PECAM-1 signaling as well as their functions in endothelial cells.
\end{abstract}

(c) 2008 Elsevier Inc. All rights reserved.

\section{Introduction}

Sphingosine 1-phosphate (S1P) is a low-molecular-weight phospholipid mainly released from stimulated platelets [1,2]. Platelet-released S1P binds to S1P receptors on surrounding endothelial cells through a paracrine route $[3,4]$. To date, five $\mathrm{S} 1 \mathrm{P}$ receptors $\left(\mathrm{S}_{1} \mathrm{P}_{1-5}\right)$ have been identified [5-10]. These S1P receptors couple to various $G$ proteins, including $G_{i}, G_{12 / 13}$, and $G_{q}[11,12]$, and activate extracellular signalregulated kinase (ERK), phospholipase C (PLC), phosphoinositide 3-kinase (PI3K), adenylyl cyclase (AC), Rho, Cdc42, and c-jun N-terminal kinase (JNK) [11-15]. In endothelial cells, S1P regulates various cellular functions, including proliferation, migration, and adhesion [16-19]. Besides, S1P also mediates re-assembly of cytoskeleton and adherens junction, as well as enhancement of endothelial barrier integrity [19-21].

Platelet/endothelial cell adhesion molecule-1 (PECAM-1) is a 130-kDa transmembrane glycoprotein expressed in endothelial cells, platelets, monocytes, and leukocytes [22,23]. In endothelial cells, PECAM-1 is mainly located at lateral cell border. Its extracellular domain forms transhomophilic interaction between adjacent cells [24-26]. Moreover,

\footnotetext{
This work was supported by grant 95-2311-B-002-018-MY2 from the National Science Council of ROC to H. Lee.

* Corresponding author. Department of Life Science, National Taiwan University, No 1, Sec. 4, Roosevelt Rd., Taipei, 10617, Taiwan, ROC. Tel.: +886 2 33662499; fax: +886 2 23636837.

E-mail address: hsinyu@ntu.edu.tw (H. Lee).
}

cytoplasmic domain of PECAM-1 contains several tyrosine residues. Upon stimulations, these tyrosine residues become phosphorylated and interact with SH2 domain-containing molecules, such as SHP-2 [27-29]. Association of SHP-2 with phosphorylated PECAM-1 was shown to stabilize adherens junctions through de-phosphorylation of beta-catenin [29-31]. Furthermore, studies on PECAM-1-deleted cells and knockout mice also indicated the association between PECAM-1 phosphorylation and endothelial permeability maintenance $[32,33]$.

Both S1P and PECAM-1 play critical roles in endothelial functions. However, the relationship between these two molecules remains unclear. In the present study, we first demonstrated that S1P-induced PECAM-1 tyrosine phosphorylation in human endothelial cells. By using pharmacological inhibitors, we demonstrated that activation of $\mathrm{G}_{\mathrm{i}}$ and Src family kinases (SFK) involves in the phosphorylation event. In addition, transfection of cSrc and Fyn siRNA attenuated S1Pinduced PECAM-1 phosphorylation in a synergistic manner. These results provide a connection between S1P and PECAM-1 signaling.

2. Materials and methods

\subsection{Reagents}

Collagenase IV, gelatin, sphingosine 1-phosphate, fatty acid-free bovine serum albumin (BSA), NP-40, horseradish peroxidase molecules (Type VI-A, 44kD), guaiacol substrate assay, and protease inhibitors (leupeptin, sodium orthovanadate, phenylmethanesulfonyl fluoride, and $\beta$-glycerophosphate) were purchased from SigmaAldrich (St. Louis, MO). PP2 and protein G plus-agarose suspension were obtained from Calbiochem (San Diego, CA). Most of the antibodies, including PECAM-1 (sc1505), cSrc (sc18), Fyn (sc16), actin (sc1616), goat anti-mouse IgG-HRP (sc2005), goat anti-rabbit 
$A$
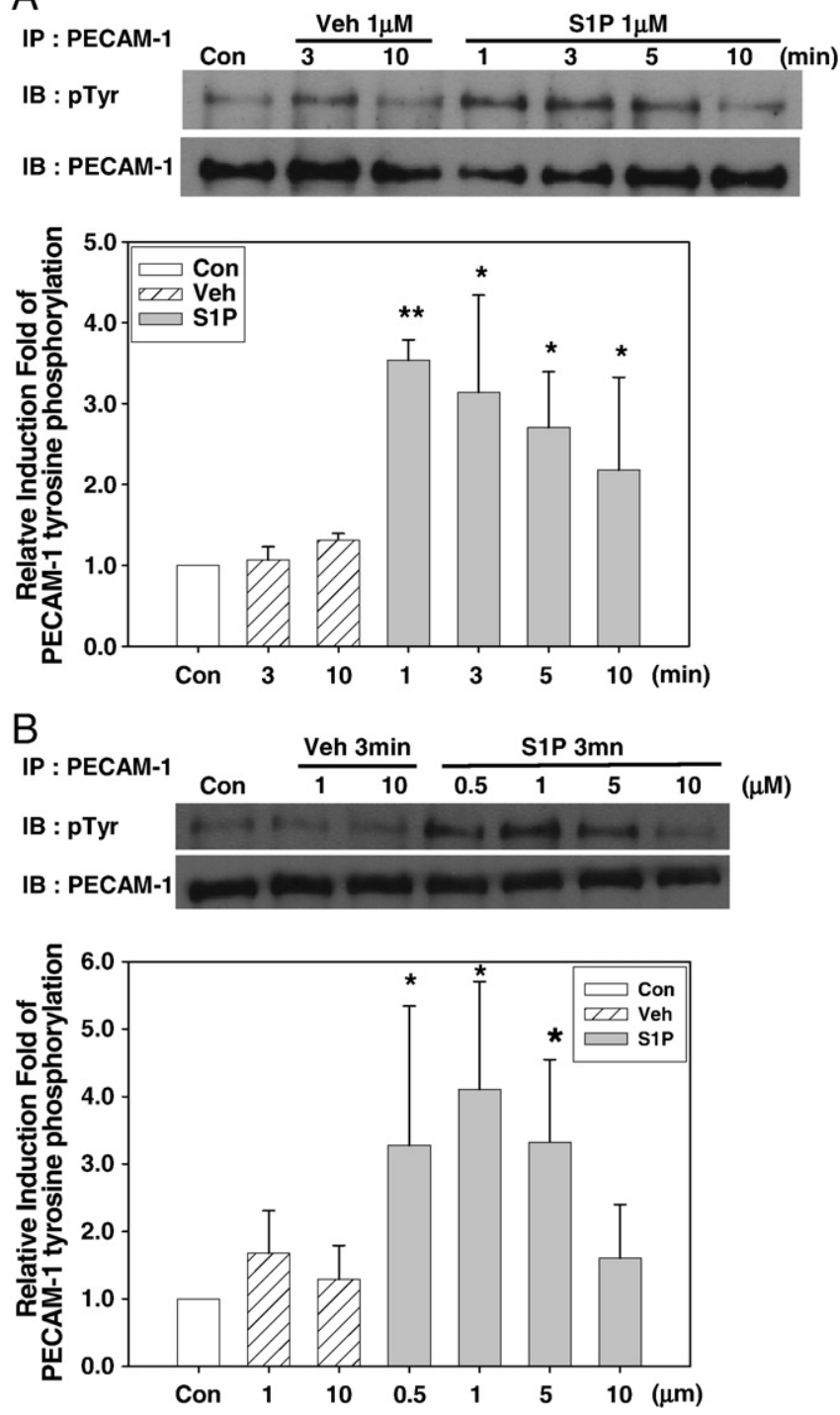

Fig. 1. S1P-induced PECAM-1 phosphorylation in a time- and concentration-dependent manner. HUVECs were treated with (A) $1 \mu \mathrm{M}$ S1P for different time periods or (B) different concentrations of S1P for 3 min. PECAM-1 phosphorylation was analyzed by immunoprecipitation (IP) and following immunoblotting (IB) with the indicated antibodies. Medium (Con) and vehicle (Veh) were used as controls. Results of one of three independent experiments are shown, and the quantified ratios of phosphorylated PECAM- 1 are presented as mean \pm SD. $\left(n=3,{ }^{*} p<0.05,{ }^{* *} p<0.01\right.$; compared with Con).

IgG-HRP (sc2004), homologous IP/WB kit ExactaCruz F (sc45043), siRNA targeting cSrc (sc44250), and Fyn (sc29321) were the products of Santa Cruz (Santa Cruz, CA). PECAM-1 antibodies for immunoprecipitation (CBL468) and immunofluorescence (555445) were respectively purchased from Chemicon (Temecula, CA) and BD Pharmingen (San Jose, CA). Phospho-tyrosine antibodies (05-321 and 610000) were respectively obtained from Upstate (Charlottesville, VA) and BD Transduction Laboratories (San Jose, CA). Src $\left[\mathrm{pY}^{418}\right]$ (44-660G) was obtained from Biosource (Camarillo, CA). The rabbit anti-goat IgG-HRP antibody (31402) and chemiluminescent substrate (34080) were purchased from Pierce (Rockford, IL). Alexa Fluor 488 donkey anti-mouse IgG (A21202) and Alexa Fluor 555 donkey anti-rabbit IgG (A31572) were the products of Molecular Probes (Eugene, OR). Endothelial growth medium (EGM), medium 199 (M199), and fetal bovine serum (FBS) were respectively purchased from Cell Applications (San Diego, CA), Biowest (Nuaillé, France), and Hyclone (Logan, UT). The kit for HUVEC electroporation (VPB-1002) was obtained from Amaxa (Gaithersburg, MD). Fluoromount-G (0100-01) was purchased from Southern Biotech (Birmingham, AL).

\subsection{HUVEC isolation}

Human umbilical cords were kindly provided by National Taiwan University Hospital (Taipei, Taiwan), under the guideline of IRB number 9561709146 of NTU hospital. Umbilical cord veins were washed twice with $1 \times$ cord buffer $(137 \mathrm{mM} \mathrm{NaCl}$, $4 \mathrm{mM} \mathrm{KCl}, 10 \mathrm{mM}$ HEPES, and $11 \mathrm{mM}$ glucose; $\mathrm{pH}$ 7.65) and incubated with $0.1 \%$ collagenase IV at $37{ }^{\circ} \mathrm{C}$ for $30 \mathrm{~min}$. After incubation, cells in collagenase were collected and cultured for the following experiments. The percentage of HUVECs to the overall cell population was examined by PECAM-1 immunostaining.

\subsection{Cell culture}

HUVECs isolated from cord veins were grown on 1\% gelatin-coated plates in M199 supplemented with $20 \%$ EGM and $10 \%$ FBS. Cells were trypsinized with $0.05 \%$ trypsinEDTA for sub-culturing. Cultures were kept at $37{ }^{\circ} \mathrm{C}$ in a humidified atmosphere with $5 \%$ $\mathrm{CO}_{2}$. All experiments were carried out with cells at passages 1 to 4 .

\subsection{S1P stimulation}

HUVECs were starved with M199 for $6 \mathrm{~h}$ before S1P treatment. S1P powder was dissolved in methanol/chloroform (9:1) to make a $1 \mathrm{mM}$ stock solution, and $0.005 \%$ fatty acid-free BSA was used as the S1P carrier in M199. The corresponding volume of methanol/chloroform with $0.005 \%$ fatty acid-free BSA was used as a vehicle control (Veh). In most experiments, HUVECs were treated with $1 \mu \mathrm{M} \mathrm{S} 1 \mathrm{P}$ at $37^{\circ} \mathrm{C}$ for $3 \mathrm{~min}$.
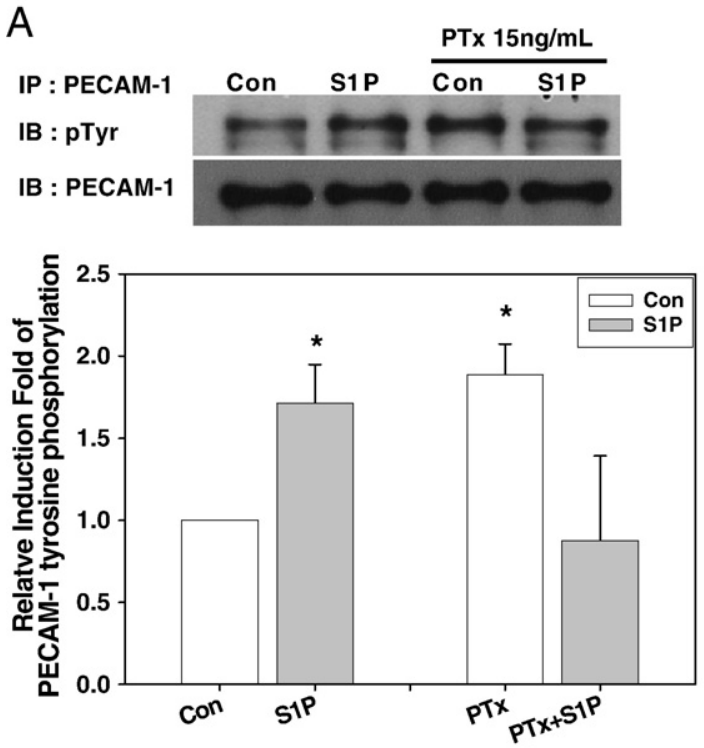

B
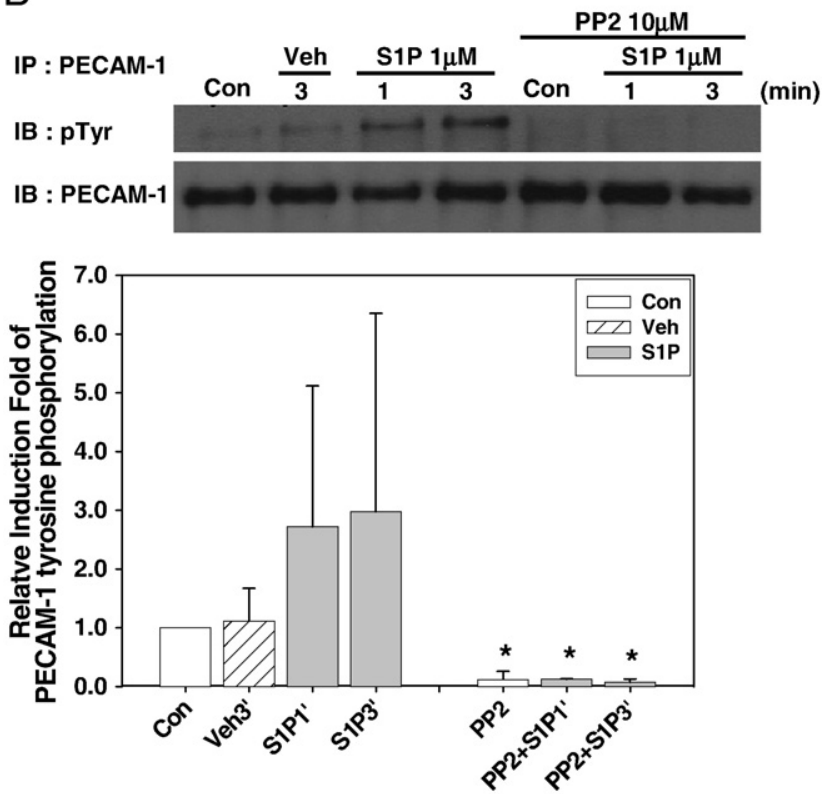

Fig. 2. S1P-induced PECAM-1 phosphorylation was mediated by $G_{i}$ and SFK. HUVECs were incubated with (A) $15 \mathrm{ng} / \mathrm{mL}$ pertussis toxin (PTx) for $16 \mathrm{~h}$ or (B) $10 \mu \mathrm{M}$ PP2 for $1 \mathrm{~h}$ before S1P treatment. PECAM-1 phosphorylation was analyzed by immunoprecipitation (IP) and following immunoblotting (IB) with the indicated antibodies. Results of one of three independent experiments are shown, and the quantified ratios of phosphorylated PECAM- 1 are presented as mean \pm SD. ( $n=3,{ }^{*} p<0.05$; compared with Con). 
A

Con Veh $\frac{\text { S1P } 1 \mu M}{1310(\min )}$

IB : Src $\left[p Y^{418}\right]$

IB : cSrc

B

Con
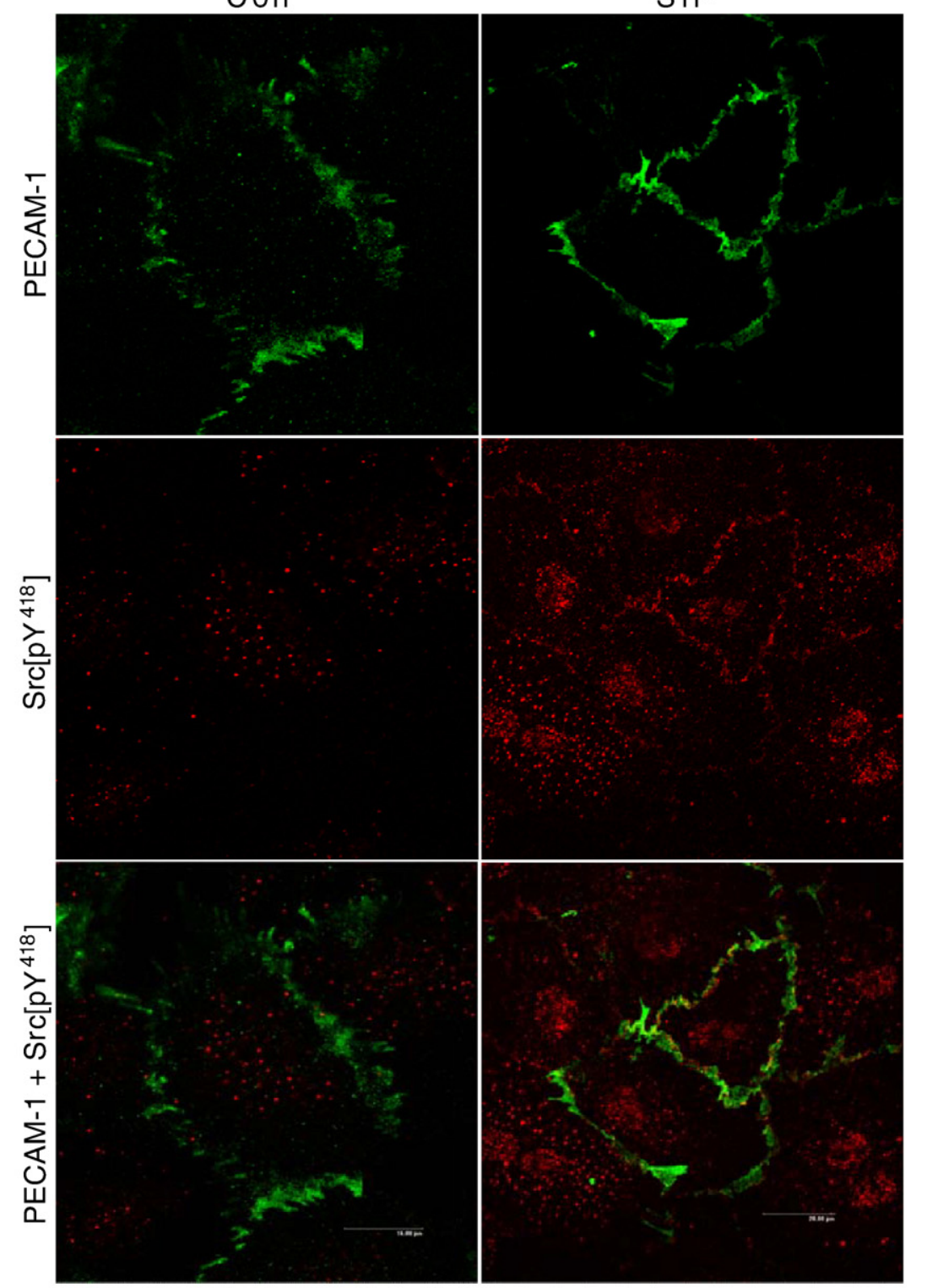

C

PTx 15ng/mL

IB : Src [pY $\left.\mathrm{P}^{418}\right]$

IB : cSrc

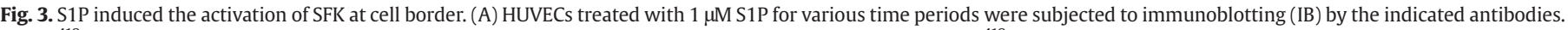

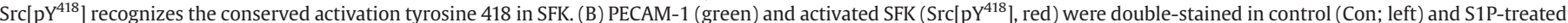

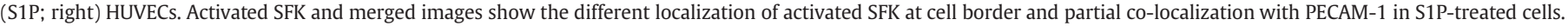

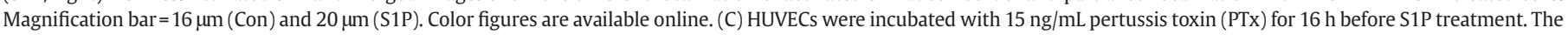
effect of S1P on SFK activation was detected by immunoblotting (IB) with the indicated antibodies. $(n=3)$.

\subsection{Immunoprecipitation}

HUVECs grown on 10-cm plates were cultured to confluent monolayers. After S1P treatment, cells were washed with cold 1× PBS and lysed with 1\% NP-40 lysis buffer
(20 mM Tris (pH 8.0), $100 \mathrm{mM} \mathrm{NaCl}, 1 \% \mathrm{NP}-40,10 \%$ glycerol, and $1 \mathrm{mM} \mathrm{Na}_{3} \mathrm{VO}_{4}$ ) containing protease inhibitors ( $10 \mu \mathrm{g} / \mathrm{mL}$ leupeptin, $2 \mathrm{mM} \mathrm{Na}_{3} \mathrm{VO}_{4}, 1 \mathrm{mM}$ PMSF, and $2 \mathrm{mM} \beta$-glycerophosphate). Lysates were collected and kept on ice. After $1 \mathrm{~h}$, lysates were centrifuged at $14,000 \mathrm{rpm}$ for $15 \mathrm{~min}$ to separate out cellular debris. 
Concentrations of clarified lysates were determined by Bradford assay (Bio-Rad). Total proteins, at 300-500 $\mu \mathrm{g}$, were incubated with $1 \mu \mathrm{g}$ IP antibodies and rotated at $4{ }^{\circ} \mathrm{C}$ overnight. The protein-antibody complexes were supplemented with $15 \mu \mathrm{L}$ of protein $\mathrm{G}$ plusagarose suspension and continually rotated at $4{ }^{\circ} \mathrm{C}$ for $5 \mathrm{~h}$. All complexes were then washed by $1 \% \mathrm{NP}-40$ lysis buffer three times, and boiled with $6 \times$ sample buffer at $95^{\circ} \mathrm{C}$ for $5 \mathrm{~min}$. The supernatants were collected for western blot.

When working on cSrc and Fyn immunoprecipitation, the rabbit homologous IP/WB kit, ExactaCruz F (Santa Cruz, sc45043), was used to reduce the disturbance of antibody heavy chain during western blot. IP antibodies and the provided matrix were mixed and continually rotated at $4{ }^{\circ} \mathrm{C}$ overnight. The mixtures were then washed with $0.5 \% \mathrm{NP}-40$ lysis buffer twice and supplemented with $500 \mu \mathrm{g}$ total proteins. Mixtures were again rotated at $4{ }^{\circ} \mathrm{C}$ overnight. The specific secondary detection reagent provided by ExactaCruz F was used when carrying out the western blot.

\subsection{Western blot}

HUVECs were lysed with $1 \%$ NP-40 lysis buffer containing protease inhibiters. Total proteins, at $20-50 \mu$, were boiled with $6 \times$ sample buffer at $95{ }^{\circ} \mathrm{C}$ for $5 \mathrm{~min}$ and resolved by $10 \%$ SDS-PAGE. Gels were transferred to PVDF membranes through a $350 \mathrm{~mA}$ current for $4 \mathrm{~h}$. Membranes were blocked with 5\% BSA in $1 \times$ TBSt (10 mM Tris, $150 \mathrm{mM} \mathrm{NaCl}$, $0.1 \%$ Tween-20; pH7.4) at room temperature for $2 \mathrm{~h}$. Primary and peroxidaseconjugated secondary antibodies were diluted to $1: 1000$ and 1:4000 in $1 \%$ BSA in 1× TBSt, incubated with membranes at room temperature for $1.5 \mathrm{~h}$. Chemiluminescent substrate was prepared, and the luminescent signal was detected according to manufacturer's instruction. Each blot was scanned, and the band density was measured by TotalLab vers. 2.01 .

\subsection{Immunofluorescence}

HUVECS grown on $1 \%$ gelatin-coated coverglasses were cultured to confluent monolayers. After S1P treatment, cells were fixed with pre-warmed $4 \%$ paraformaldehyde at room temperature for $30 \mathrm{~min}$, followed by two rinses with $1 \times$ PBS. Cells were then blocked and permeabilized with blocking solution (0.5\% BSA and 0.1\% Triton X-100 in $1 \times$ PBS) at room temperature for $30 \mathrm{~min}$. Primary and Alexa Fluor-conjugated secondary antibodies were diluted to $1: 100$ and 1:500 in blocking solution, respectively incubated with cells at $4{ }^{\circ} \mathrm{C}$ overnight and $37^{\circ} \mathrm{C}$ for $2 \mathrm{~h}$. Coverglasses were washed twice with $1 \times$ PBS between the incubation of the primary and secondary antibodies. After secondary antibody incubation, the coverglasses were thoroughly washed with distilled water and mounted by Fluoromount-G. Fluorescence was then detected by confocal microscopy.

\subsection{RNA interference and electroporation}

The cSrc and Fyn siRNAs purchased from Santa Cruz were dissolved in $180 \mu \mathrm{L}$ siRNA diluent to make a solution of $1.28 \mu \mathrm{g}$ siRNA in $5 \mu \mathrm{L}$. The Amaxa Nucleofector biosystems with the HUVEC-specific U-01 program was used for siRNA transfection. HUVECs between passages 1 to 3 were grown to $95 \%$ confluence and trypsinized to determine cell numbers. According to manufacturer's instructions, each reaction for siRNA electroporation contained $10^{6}$ cells and $2.56 \mu \mathrm{g}$ siRNA in $100 \mu \mathrm{L}$ of HUVEC Nucleofector solution. When working on cSrc and Fyn double-knockdown, the amount of each siRNA was reduced to $1.28 \mu \mathrm{g}$ to achieve the same total volume. After electroporation, the transfected cells were cultured at $37^{\circ} \mathrm{C}$ for $42 \mathrm{~h}$, followed by $6 \mathrm{~h}$ of starvation. Cells were then ready for S1P treatment.

\subsection{HUVEC monolayer permeability assay}

HUVECs were cultured in trans-well chambers $(0.4 \mu \mathrm{m}$ pore-size polycarbonate filters; Costar, Cambridge, Mass., USA). After reaching confluence, the cells were washed, and then the medium was replaced with the indicated conditions $(0.3 \mathrm{~mL}$ in the upper chamber and $1 \mathrm{~mL}$ in the lower chamber). Horseradish peroxidase molecules at a concentration of $0.126 \mu \mathrm{M}$ were added to the upper compartment. After incubation for $1 \mathrm{~h}$, the medium in the lower compartment was assayed for enzymatic activity using a photometric guaiacol substrate assay.

\subsection{Statistical analysis}

Each result obtained from at least three independent experiments is expressed as mean \pm SD. Data were analyzed by ANOVA, followed by Fisher's protected leastsignificant difference test (Statview 5.0; Abacus Concept, Berkeley, CA). $p<0.05$ was considered statistically significant.

\section{Results}

\subsection{S1P-induced PECAM-1 phosphorylation in a time- and concentration-} dependent manner

First, we investigated the effect of S1P on PECAM-1 phosphorylation. HUVECs were starved and treated with $1 \mu \mathrm{MS} 1 \mathrm{P}$ for different time periods. Compared with medium (Con) and vehicle control (Veh), the ratio of phosphotyrosine to total PECAM-1 peaked at 1 to $3 \mathrm{~min}$, and returned to basal level at 10 min of S1P treatment (Fig. 1A). To further confirm S1Pinduced phosphorylation, HUVECs were treated with various concentrations of S1P. As in Fig. 1B, PECAM-1 tyrosine phosphorylation was increased at $0.5 \mu \mathrm{M}$, peaked at $1 \mu \mathrm{M}$, and decreased from 5 to $10 \mu \mathrm{M}$ of S1P treatments. These results indicated that S1P-induced PECAM-1 phosphorylation in a time- and dosage-dependent manner.
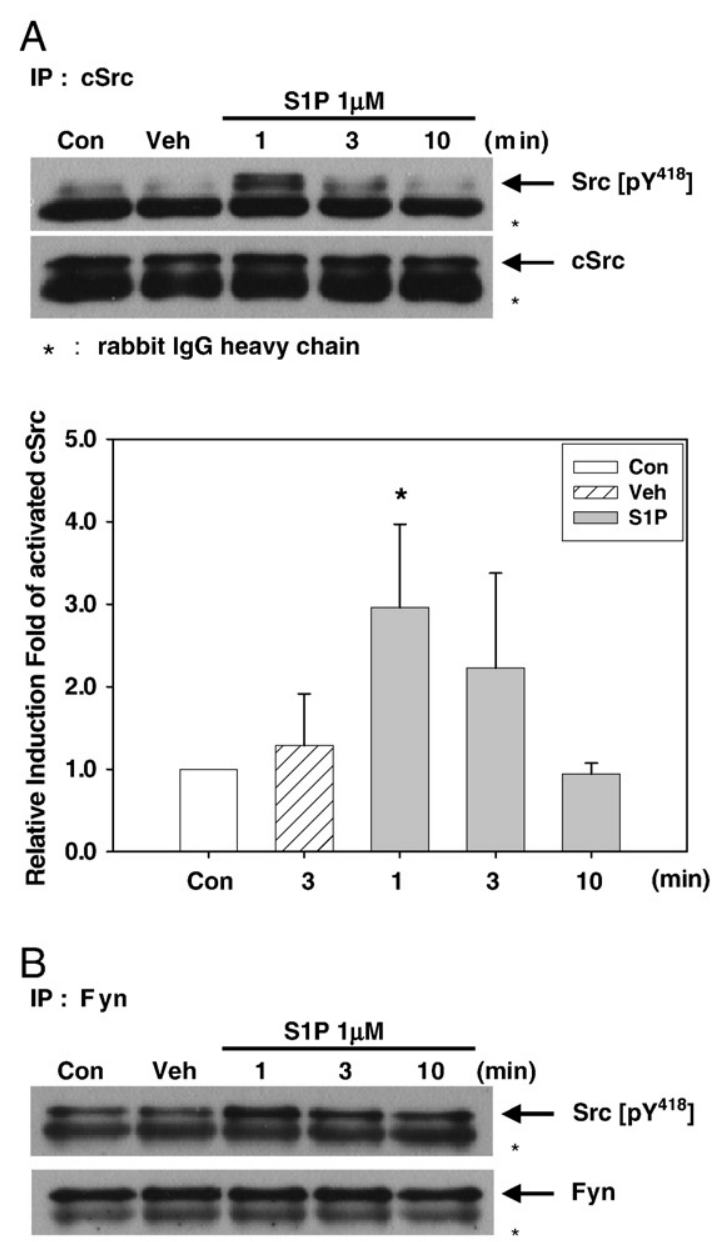

* : rabbit IgG heavy chain

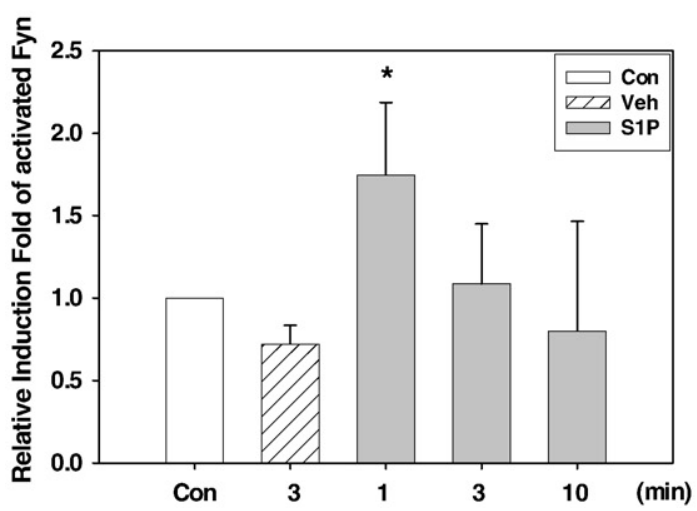

Fig. 4. S1P-induced cSrc and Fyn activation in HUVECs. HUVECs treated with $1 \mu \mathrm{M}$ S1P for various time periods were subjected to (A) cSrc and (B) Fyn immunoprecipitation (IP), followed by immunoblotting (IB) with the indicated antibodies. Ratios of activated form $\left(\mathrm{Src}\left[\mathrm{pY}^{418}\right]\right)$ to total precipitated kinases represent the effect of S1P on cSrc and Fyn activation. The asterisk $\left({ }^{*}\right)$ indicates the rabbit IgG heavy chain. $\left(n=3,{ }^{*} p<0.05\right.$; compared with Con). 
A

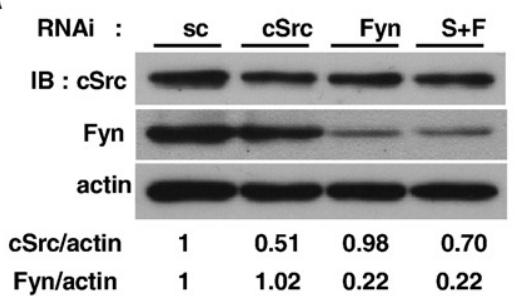

B
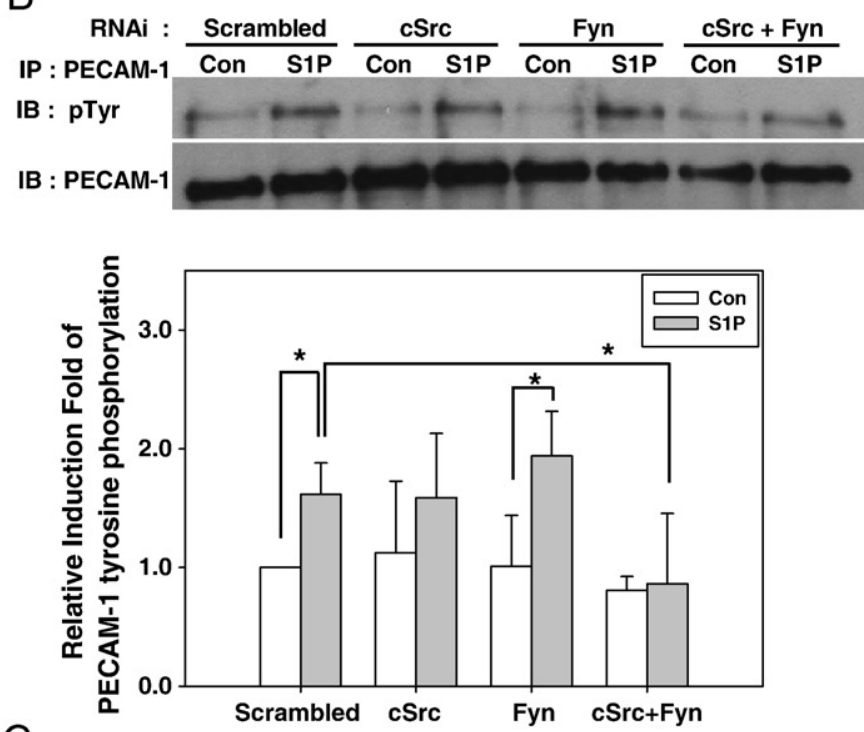

C
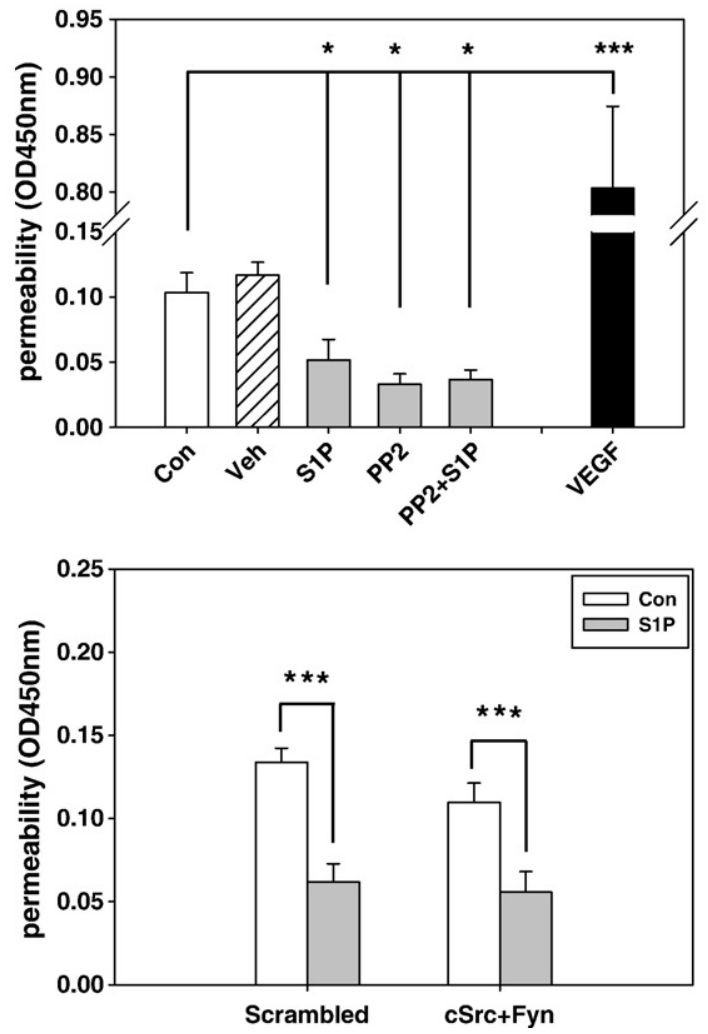

3.2. S1P-induced PECAM-1 phosphorylation was mediated through $G_{i}$ and $S F K$

After confirming the effect of S1P on PECAM-1 phosphorylation, we used pharmacological inhibitors to clarify the involved signaling pathway. $G_{i}$ is one of the major $G$ proteins which couple to S1P receptors $[11,12]$. Pertussis toxin (PTx), a frequently used $G_{i}$ inhibitor for S1P receptor signaling, was pretreated to HUVECs for $16 \mathrm{~h}$. As the result, S1Pinduced PECAM-1 phosphorylation was significantly suppressed by PTx (Fig. 2A). In PTx-treated cells, PECAM-1 tyrosine phosphorylation was increased when compared with untreated cells, which might be due to long-term PTx pretreatment-caused cell death and disruption of cellcell interactions [34]. Moreover, one-hour pretreatment of PP2, a selected SFK inhibitor, also markedly inhibited PECAM-1 phosphorylation (Fig. 2B). These results indicated that S1P induces PECAM-1 phosphorylation through a $\mathrm{G}_{\mathrm{i}}{ }^{-}$and SFK-dependent pathway.

\subsection{S1P induced the activation of SFK at cell border}

Inhibition by PP2 suggested the participation of SFK in S1P-induced PECAM-1 phosphorylation. Therefore, we further examined the effect of S1P on SFK activation. After S1P treatment, activated SFK $\left(\operatorname{Src}\left[\mathrm{pY}^{418}\right]\right)$ and cSrc were separately blotted. The amount of Src $\left[\mathrm{pY}^{418}\right]$ significantly increased within $1 \mathrm{~min}$ (Fig. 3A). Moreover, double-staining of PECAM-1 (green) and $\mathrm{Src}\left[\mathrm{pY}^{418}\right]$ (red) was carried out (color figures are available online). Compared with control, S1P significantly triggered the localization of activated SFK to cell border. Merged images at the bottom showed partial co-localization of activated SFK with PECAM-1 after S1P treatment (Fig. 3B).

In Fig. 2A, the inhibition of S1P-induced PECAM-1 phosphorylation by PTx indicated the participation of $G_{i j}$. Therefore, the effect of PTx on SFK activation was examined. Pretreatment with PTx efficiently blocked S1P-induced activation of SFK (Fig. 3C), which strongly suggested that S1P-induced PECAM-1 phosphorylation is mediated through $G_{i}$ and subsequent SFK.

Expression patterns of SFK members vary in different cell types [35]. In endothelial cells, the major SFK expressed are cSrc, Fyn, and Yes [35]. Moreover, PP2 is known to inhibit activities of cSrc, Fyn, Lck, and Hck [36]. These results suggested that cSrc and Fyn might be involved in S1P-induced PECAM-1 phosphorylation. Thus, the activities of immunoprecipitated cSrc and Fyn were detected by $\operatorname{Src}\left[\mathrm{pY}^{418}\right]$ antibody. The results showed that both cSrc (Fig. 4A) and Fyn (Fig. 4B) were activated by S1P treatment within 1 min, which was similar to Fig. 3A.

\subsection{S1P-induced PECAM-1 phosphorylation through both cSrc and Fyn}

To identify whether cSrc or Fyn was responsible for S1P-induced PECAM-1 phosphorylation, specific siRNAs were electroporated into HUVECs, and the RNAi efficiency was detected by western blot. Compared to scrambled siRNA control (sc), RNAi efficiencies of cSrc and Fyn single-knockdown were $49 \%$ and $78 \%$, respectively. In cSrc/Fyn double-knockdown $(\mathrm{S}+\mathrm{F})$, the remaining protein expressions of cSrc and Fyn were $70 \%$ and $22 \%$ (Fig. 5A).

Fig. 5. S1P-induced PECAM-1 phosphorylation through cSrc and Fyn. (A) Scrambled (sc), cSrc, Fyn, cSrc+Fyn (S+F) siRNA was transfected into HUVECs. Forty-eight hours after transfection, targeted kinases were immunoblotted (IB) with the indicated antibodies. The bottom numbers for the protein expression levels represented the RNAi efficiency. (B) Scrambled, cSrc, Fyn, and cSrc+Fyn siRNA transfected HUVECs were treated with medium (Con) or $1 \mu \mathrm{M} \mathrm{S1P}(\mathrm{S} 1 \mathrm{P})$ for 3 min and subjected to PECAM-1 immunoprecipitation (IP), followed by immunoblotting (IB) with the indicated antibodies. Results of one of four independent results are shown. Phosphorylated PECAM-1 ratios compared with scrambled control are shown as mean \pm SD. $(n=4$, ${ }^{*} p<0.05$ ) (C) HUVEC monolayer permeability with indicated treatment was measured by photometric assay and is shown as mean $\pm \operatorname{SD}\left(n=3,{ }^{*} p<0.05\right.$; compared with Con). Con: medium only; Veh: vehicle medium; VEGF was used as positive control for disruption of monolayer integrity. (D) Scrambled and cSrc+Fyn siRNA transfected HUVECs assayed for monolayer permeability is shown as mean $\pm \operatorname{SD}\left(n=4,{ }^{* * *} p<0.001\right)$. 
Cells transfected with siRNA were then treated with $1 \mu \mathrm{M}$ of S1P to examine PECAM-1 phosphorylation. Compared with scrambled siRNA transfectant, neither cSrc nor Fyn single-knockdown caused significant inhibition of S1P-induced PECAM-1 phosphorylation. However, cSrc/Fyn double-knockdown showed significant decrease in S1Pelicited PECAM-1 phosphorylation (Fig. 5B). These results implied that both cSrc and Fyn contribute to this S1P-induced PECAM- 1 tyrosine phosphorylation in human endothelial cells.

\subsection{S1P decreased endothelial monolayer permeability}

To further assess the effect of S1P-induced PECAM-1 phosphorylation on endothelial barrier integrity, trans-well permeability assay was conducted. VEGF was used as positive control for disruption of monolayer integrity. Compared with medium (Con) and vehicle control (Veh), S1P significantly decreased HUVEC monolayer permeability (Fig. 5C). However, the permeability was also decreased by pretreatment of PP2, and the pretreatment of PP2 did not inhibit the effect of S1P on endothelial permeability. Similar result was obtained in cSrc/Fyn double-knockdowned cells. The endothelial permeability was slightly declined in double-knockdown cells, and the S1P effect on permeability was not affected (Fig. 5D). Collectively, these evidences indicated that S1P-enhanced barrier integrity might not be regulated by PECAM- 1 tyrosine phosphorylation.

\section{Discussion}

It is suggested that PECAM-1 could coordinate S1P signaling through recruitment of different $G_{\alpha}$ proteins in lipid rafts [37]. Herein, we demonstrated that PECAM-1 is a downstream target for S1P signaling. In this study, we clarified for the first time that S1P induces PECAM-1 tyrosine phosphorylation in human endothelial cells. In addition, we also delineated that $\mathrm{G}_{\mathrm{i}}$ and SFK activations are involved in PECAM-1 tyrosine phosphorylation.

Recent reports suggested that tyrosine 663 of PECAM-1 was prominent for its localization in sub-membrane microdomains and monocyte transendothelial migration $[38,39]$, which indicates that PECAM-1 tyrosine phosphorylation and its functional regulation might occur in specific membrane domains. In Fig. 3B, S1P-activated SFK showed only partial co-localization with PECAM-1. It was possibly due to restrictive occurrence in specialized membrane sub-domains. In addition, previous studies on sphingosine kinase-1 (SPhK-1) showed its interaction with PECAM-1 by using in vitro binding assay and co-immunoprecipitation in SPhK-1-overexpressed endothelial cells. However, failure in conducting co-immunoprecipitation in primarily cultured HUVEC suggested that the interaction might be extremely transient $[34,40]$. Therefore, the partial co-localization of activated SFK and PECAM-1 in our experiments might also be caused by transient interaction between these two molecules.

Apparently, phosphorylation of PECAM-1 cytoplasmic tyrosines depends on intracellular tyrosine kinases. Evidence from in vitro binding assay, over-expression, expression cloning and gene silencing approaches showed SFK [27,41-44], Csk [43] and Fer [45] were all capable of catalyzing PECAM-1 phosphorylation, depending on both applying stimulation and cell types. In this study, we first demonstrated that S1P-induced PECAM-1 tyrosine phosphorylation in primarily cultured HUVEC is mediated synergistically through cSrc and Fyn.

Results from cSrc and Fyn siRNA co-transfection experiments demonstrated that the two kinases mediated S1P-induced PECAM-1 phosphorylation in a synergistic manner (Fig. 5B). Previous reports showed that the compensatory effects among SFK members occur in various aspects, including expression patterns, cellular distributions, and association partners. In addition, abnormal phenotypes observed in double- or triple-kinase knockout mice are also more severe than in single-kinase knockout mice $[35,46]$. These compen- satory effects therefore correspond with our finding that S1Pinduced PECAM-1 phosphorylation is only suppressed in doubleknockdowned HUVECs.

In Fig. 5C, S1P decreased the amount of permeating horseradish peroxidase molecules in our experiment. Similar barrier-protection effect of S1P was also found in pulmonary artery and lung microvascular endothelial cells [20]. It is suggested that S1P improved endothelial barrier integrity through modulating adherens junction and tight junction assembly [21,47]. However, the role of PECAM-1 in S1P-regulated barrier function is seldom mentioned. As Biswas et al. suggested, SHP-2 and beta-catenin interact with phosphorylated PECAM-1 [33]. Formation of this tri-molecular complex facilitates beta-catenin de-phosphorylation by SHP-2, which stabilizes the VEcadherin/cytoskeleton association at adherens junction, and causes faster recovery of barrier integrity [32,33]. However, pretreatment of PP2 and cSrc/Fyn double-knockdown also decreased the endothelial permeability. Because SFK is able to bind and control phosphorylation status of VE-cadherin [48,49], abrogation of SFK activation may cause the stabilization of adherens junctions, thereby reduces trans-well permeability. Moreover, PP2 pretreatment and cSrc/Fyn RNAi did not block the effect of S1P on endothelial permeability. These results suggested that the S1P effect on permeability of endothelial barriers might not be regulated through phosphorylation of PECAM-1.

In summary, we first demonstrated that S1P-induced PECAM-1 tyrosine phosphorylation through both cSrc and Fyn. The mechanism of S1P-induced PECAM-1 phosphorylation also provides a linkage between S1P and PECAM-1 signaling in human endothelial cells.

\section{Acknowledgement}

We thank Dr. F. J. Hsieh at the National Taiwan University Hospital for providing umbilical cords for endothelial cell preparation (IRB 9561709146).

\section{References}

[1] Y. Yatomi, F. Ruan, S. Hakomori, Y. Igarashi, Blood 86 (1) (1995) 193-202.

[2] Y. Yatomi, Y. Ozaki, T. Ohmori, Y. Igarashi, Prostaglandins 64 (1-4) (2001) 107-122.

[3] H. Rosen, E.J. Goetzl, Nat. Rev. Immunol. 5 (7) (2005) 560-570.

[4] Y. Yatomi, T. Ohmori, G. Rile, F. Kazama, H. Okamoto, T. Sano, K. Satoh, S. Kume, G. Tigyi, Y. Igarashi, Y. Ozaki, Blood 96 (10) (2000) 3431-3438.

[5] T. Hla, T. Maciag, J. Biol. Chem. 265 (16) (1990) 9308-9313.

[6] H. Okazaki, N. Ishizaka, T. Sakurai, K. Kurokawa, K. Goto, M. Kumada, Y. Takuwa, Biochem. Biophys. Res. Commun. 190 (3) (1993) 1104-1109.

[7] A.J. MacLennan, C.S. Browe, A.A. Gaskin, D.C. Lado, G. Shaw, Mol. Cell. Neurosci. 5 (3) (1994) 201-209.

[8] F. Yamaguchi, M. Tokuda, O. Hatase, S. Brenner, Biochem. Biophys. Res. Commun. 227 (2) (1996) 608-614.

[9] M.H. Graler, G. Bernhardt, M. Lipp, Genomics 53 (2) (1998) 164-169.

[10] D.S. Im, C.E. Heise, N. Ancellin, B.F. O'Dowd, G.J. Shei, R.P. Heavens, M.R. Rigby, T. Hla, S. Mandala, G. McAllister, S.R. George, K.R. Lynch, J. Biol. Chem. 275 (19) (2000) 14281-14286.

[11] T. Sanchez, T. Hla, J. Cell. Biochem. 92 (5) (2004) 913-922.

[12] B. Anliker, J. Chun, Semin. Cell Dev. Biol. 15 (5) (2004) 457-465.

[13] S. An, E.J. Goetzl, H. Lee, J. Cell Biochem. Suppl. 30-31 (1998) 147-157.

[14] B. Anliker, J. Chun, J. Biol. Chem. 279 (20) (2004) 20555-20558.

[15] Y. Yatomi, Curr. Pharm. Des. 12 (5) (2006) 575-587.

[16] F. Wang, J.R. Van Brocklyn, J.P. Hobson, S. Movafagh, Z. Zukowska-Grojec, S. Milstien, S. Spiegel, J. Biol. Chem. 274 (50) (1999) 35343-35350.

[17] H. Lee, E.J. Goetzl, S. An, Am. J. Physiol. Cell Physiol. 278 (3) (2000) 612-618.

[18] H. Zhang, N.N. Desai, A. Olivera, T. Seki, G. Brooker, S. Spiegel, J. Cell. Biol. 114 (1) (1991) 155-167.

[19] Y. Shikata, K.G. Birukov, J.G. Garcia, J. Appl. Physiol. 94 (3) (2003) 1193-1203.

[20] J.G. Garcia, F. Liu, A.D. Verin, A. Birukova, M.A. Dechert, W.T. Gerthoffer, J.R. Bamberg, D. English, J. Clin. Invest. 108 (5) (2001) 689-701.

[21] M.J. Lee, S. Thangada, K.P. Claffey, N. Ancellin, C.H. Liu, M. Kluk, M. Volpi, R.I. Sha'afi, T. Hla, Cell 99 (3) (1999) 301-312.

[22] P.J. Newman, M.C. Berndt, J. Gorski, G.C. White 2nd, S. Lyman, C. Paddock, W.A. Muller, Science 247 (4947) (1990) 1219-1222.

[23] A.M. Muller, M.I. Hermanns, C. Skrzynski, M. Nesslinger, K.M. Muller, C.J. Kirkpatrick, Exp. Mol. Pathol. 72 (3) (2002) 221-229.

[24] Q.H. Sun, H.M. DeLisser, M.M.Zukowski, C. Paddock, S.M. Albelda, P.J. Newman, J. Biol, Chem. 271 (19) (1996) 11090-11098.

[25] J. Sun, J. Williams, H.C. Yan, K.M. Amin, S.M. Albelda, H.M. DeLisser, J. Biol. Chem. 271 (31) (1996) 18561-18570. 
[26] J.P. Newton, C.D. Buckley, E.Y. Jones, D.L. Simmons, J. Biol. Chem. 272 (33) (1997) 20555-20563.

[27] T.T. Lu, M. Barreuther, S. Davis, J.A. Madri, J. Biol. Chem. 272 (22) (1997) 14442-14446.

[28] P.J. Newman, D.K. Newman, Arterioscler. Thromb. Vasc. Biol. 23 (6) (2003) 953-964

[29] N. Ilan, J.A. Madri, Curr. Opin. Cell Biol. 15 (5) (2003) 515-524.

[30] D. Gratzinger, M. Barreuther, J.A. Madri, Biochem. Biophys. Res. Commun. 301 (1) (2003) 243-249.

[31] N. Ilan, S. Mahooti, D.L. Rimm, J.A. Madri, J. Cell Sci. 112 (Pt 18) (1999) 3005-3014.

[32] D. Graesser, A. Solowiej, M. Bruckner, E. Osterweil, A. Juedes, S. Davis, N.H. Ruddle B. Engelhardt, J.A. Madri, J. Clin. Invest. 109 (3) (2002) 383-392.

[33] P. Biswas, S. Canosa, D. Schoenfeld, J. Schoenfeld, P. Li, L.C. Cheas, J. Zhang, A. Cordova, B. Sumpio, J.A. Madri, Am. J. Pathol. 169 (1) (2006) 314-324.

[34] V. Limaye, X. Li, C. Hahn, P. Xia, M.C. Berndt, M.A. Vadas, J.R. Gamble, Blood 105 (8) (2005) 3169-3177.

[35] C.A. Lowell, P. Soriano, Genes Dev. 10 (15) (1996) 1845-1857.

[36] J.H. Hanke, J.P. Gardner, R.L. Dow, P.S. Changelian, W.H. Brissette, E.J. Weringer, B.A. Pollok, P.A. Connelly, J. Biol. Chem. 271 (2) (1996) 695-701.

[37] D. Gratzinger, S. Canosa, B. Engelhardt, J.A. Madri, Faseb J. 17 (11) (2003) 1458-1469.

[38] B. Dasgupta, Z. Mamdouh, W.A. Muller, Experimental Biology Annual Meeting, 2007, Washinton, DC, Faseb J., vol. 21, 2007, p. 248.9, 21.
[39] Z. Mamdouh, X. Chen, L.M. Pierini, F.R. Maxfield, W.A. Muller, Nature 421 (6924) (2003) 748-753.

[40] Y. Fukuda, Y. Aoyama, A. Wada, Y. Igarashi, Biochim. Biophys. Acta 1636 (1) (2004) $12-21$.

[41] D.K. Newman, C. Hamilton, P.J. Newman, Blood 97 (8) (2001) 2351-2357.

[42] M. Osawa, M. Masuda, N. Harada, R.B. Lopes, K. Fujiwara, Eur. J. Cell Biol. 72 (3) 1997) 229-237.

[43] M.Y. Cao, M. Huber, N. Beauchemin, J. Famiglietti, S.M. Albelda, A. Veillette, J. Biol. Chem. 273 (25) (1998) 15765-15772.

[44] M. Cicmil, J.M. Thomas, T. Sage, F.A. Barry, M. Leduc, C. Bon, J.M. Gibbins, J. Biol. Chem. 275 (35) (2000) 27339-27347.

[45] N. Kogata, M. Masuda, Y. Kamioka, A. Yamagishi, A. Endo, M. Okada, N. Mochizuki, Mol. Biol. Cell 14 (9) (2003) 3553-3564.

[46] P.L. Stein, H. Vogel, P. Soriano, Genes Dev. 8 (17) (1994) 1999-2007.

[47] J.F. Lee, Q Zeng, H. Ozaki, L. Wang, A.R. Hand, T. Hla, E. Wang, M.J. Lee, J. Biol. Chem. 281 (39) (2006) 29190-29200.

[48] Y. Wallez, I. Vilgrain, P. Huber, Trends. Cardiovasc. Med. 16 (2) (2006) 55-59.

[49] Y. Wallez, F. Cand, F. Cruzalegui, C. Wernstedt, S. Souchelnytskyi, I. Vilgrain, P. Huber, Oncogene 26 (7) (2007) 1067-1077. 